\title{
Robotics and neuroscience: A rhythmic interaction
}

\author{
Renaud Ronsse $^{\mathrm{a}}$, Philippe Lefèvre ${ }^{\mathrm{b}, \mathrm{c}}$, Rodolphe Sepulchre ${ }^{\mathrm{a}, *}$ \\ a Department of Electrical Engineering and Computer Science (Montefiore Institute), Université de Liège, Grande Traverse 10 (B28), B-4000 Liège, Belgium \\ ${ }^{\mathrm{b}}$ Centre for Systems Engineering and Applied Mechanics (CESAME), Université catholique de Louvain, Avenue Georges Lemaître 4, B-1348 Louvain-la-Neuve, Belgium \\ ${ }^{\mathrm{c}}$ Laboratory of Neurophysiology, Université catholique de Louvain, Avenue Hippocrate 54, B-1200 Bruxelles, Belgium
}

\section{A R T I C L E I N F O}

Article history:

Received 19 December 2006

Received in revised form

7 March 2008

Accepted 7 March 2008

Keywords:

Rhythmic control

Impact juggling

Sensory feedback

Robustness

Performance

Movement primitives

\begin{abstract}
A B S T R A C T
At the crossing between motor control neuroscience and robotics system theory, the paper presents a rhythmic experiment that is amenable both to handy laboratory implementation and simple mathematical modeling. The experiment is based on an impact juggling task, requiring the coordination of two upper-limb effectors and some phase-locking with the trajectories of one or several juggled objects. We describe the experiment, its implementation and the mathematical model used for the analysis. Our underlying research focuses on the role of sensory feedback in rhythmic tasks. In a robotic implementation of our experiment, we study the minimum feedback that is required to achieve robust control. A limited source of feedback, measuring only the impact times, is shown to give promising results. A second field of investigation concerns the human behavior in the same impact juggling task. We study how a variation of the tempo induces a transition between two distinct control strategies with different sensory feedback requirements. Analogies and differences between the robotic and human behaviors are obviously of high relevance in such a flexible setup.
\end{abstract}

(c) 2008 Elsevier Ltd. All rights reserved.

\section{Introduction}

System-theoretic concepts and related engineering computational tools are increasingly used in the emerging discipline of computational motor control (Jordan \& Wolpert, 1999; Scott \& Norman, 2003; Shadmehr \& Wise, 2005; Wolpert \& Ghahramani, 2000). The most celebrated concepts include the so-called internal model principle (see e.g. Haruno, Wolpert, and Kawato (2001), Kawato (1999), Mehta and Schaal (2002), Miall, Weir, Wolpert, and Stein (1993), Wolpert and Miall (1996) and Wolpert, Miall, and Kawato (1998)) and the theory of optimal control (see e.g. Scott (2004), Todorov (2004) and Todorov and Jordan (2002)). The existence of these underlying principles in the brain has been demonstrated at least (a) to precompute trajectories, (b) to compute the motor command (muscles actuation through inverse dynamics) given the expected trajectory, and (c) to make on-line feedback corrections (closedloop control).

Computational questions in human motor control often parallels questions in robotics (Schaal \& Schweighofer, 2005). The implementation of complex control architectures in the human brain indeed provides a source of inspiration in robotics designs. Conversely, the computational and system-theoretic models in

\footnotetext{
* Corresponding author. Tel.: +32 436629 87; fax: +32 43662989 .

E-mail address: R.Sepulchre@ulg.ac.be (R. Sepulchre).
}

robotics provide useful insights into the interpretation of the highdimensional behavioral and neurophysiological data sets.

In this paper, we describe a rhythmic experiment developed by the authors at the intersection between behavioral neuroscience and robotics studies. We use this experiment to study the role of sensory feedback in rhythmic tasks, from a system-theoretic and experimental perspective, both in robotics and in human behavior. By definition, rhythmic movements are not sequential and are continuous in time. However, rhythmic tasks often require the recruitment of simultaneous degrees of freedom, hence requiring movement coordination (Bernstein, 1967; Kelso, 1995; Kelso, Southard, \& Goodman, 1979; Turvey, 1990) between several limbs. Inter-limb coordination rules underlie the possible movement patterns, and those principles cannot be inferred from the laws of single-joint or single-limb movements (Swinnen, 2002; Swinnen \& Wenderoth, 2004). At the frequency level, the default mode of coordination is synchronization, ubiquitous in biological systems (the recent book by Strogatz (2003), abounds with such examples). The phase relationships between the oscillating limbs are also governed by coordination rules. As an example, the finger tapping task introduced by Kelso (1984) is stabilized mainly through two phase-locking modes: in-phase (homologous activations) and anti-phase (180 deg of lag in activations). Moreover, beyond a certain movement frequency threshold, the anti-phase stability vanishes, revealing that the in-phase mode is in fact more stable and less attention demanding (Kelso, 1995). This coordination task is recognized as a benchmark example in the motor control literature with bifurcations in the stability 


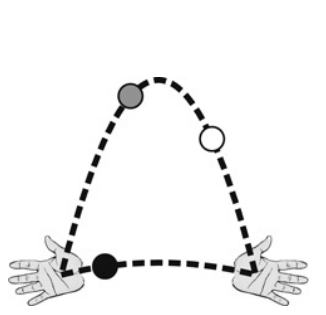

(a) The shower.

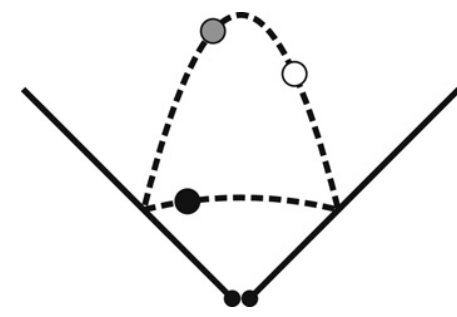

(b) Period-two

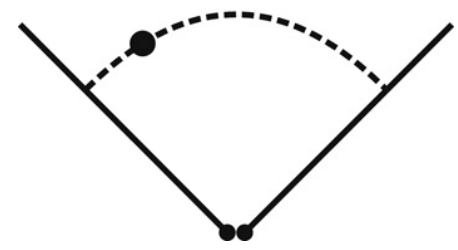

(c) Period-one.

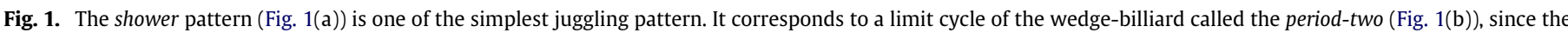

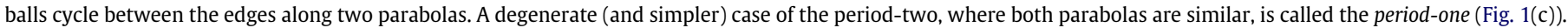

diagram of cyclical patterns. Switching between different gaits in animal behavior, as the movement frequency changes, is another example (see e.g. Collins and Richmond (1994)). In the task described in the present paper, we will see that several modes of coordination exist as well, and that their recruitment depends on the task context.

Rhythmicity potentially simplifies the execution of a given task. It corresponds to a steady-state actuation, depending on few parameters such as frequency and amplitude, in contrast with transient trajectories that typically require sustained planning and feedback. As a consequence, rhythmic movements do not necessarily recruit the same neural circuitries than discrete movements. This has been suggested by recent behavioral (Buchanan, Park, \& Shea, 2006; Sternad, Dean, \& Schaal, 2000; van Mourik \& Beek, 2004) and imaging (Schaal, Sternad, Osu, \& Kawato, 2004) studies. While rhythmic movements can be programmed by low level Central Pattern Generators (CPGs) (see e.g. Cohen, Rossignol, and Grillner (1988), Duysens and Van de Crommert (1998) and Swinnen (2002)), discrete movements also recruit higher cortical areas, that have been shown to play a role in the processing of sensory feedback (Desmurget et al., 2001). The level of feedback processing thus appears to be different in both kinds of movements. Learning obviously plays an important role in that distinction. Recently, Puttemans, Wenderoth, and Swinnen (2005) studied a complex bimanual coordination task through the course of the different learning phases. Their study evidenced that the neural network involved in the rhythmic task progressively switches from a highly "attention demanding sensory processing" (activating more cortical areas) to an "automatized" control as the learning progresses. This underlies the relevance of control strategies resting mainly on open-loop control.

Previous studies in the literature underlined the importance of open-loop strategies in rhythmic tasks. Sternad, Schaal and coworkers studied the 1D bounce juggling task and showed that the steady-state racket trajectory is similar to an open-loop harmonic movement (see e.g. Schaal, Atkeson, and Sternad (1996), Sternad, Duarte, Katsumata, and Schaal (2001a, 2001b)). In the context of rhythmic locomotion, passive strategies have inspired passive-based designs with small active power sources to walk on level ground, revealing the prominence of passive stability in the architecture of the human locomotor system (see e.g. Collins, Ruina, Tedrake, and Wisse (2005), Collins, Wisse, and Ruina (2001) and Kuo (1999)).

The paper is organized as follows. The experimental setup, its robotic implementation and mathematical model are presented in Section 2. In Section 3, we discuss the role of feedback to stabilize some rhythmic periodic orbits. We show how limited feedback can be added to the open-loop control to make a robust closed-loop design based on limited sensing. In Section 4, we illustrate typical behavior of human subjects in our impact juggling experiment at different tempi, showing a transition from "intermittent planning" strategies to rhythmic strategies, with a decrease in the required feedback demand.

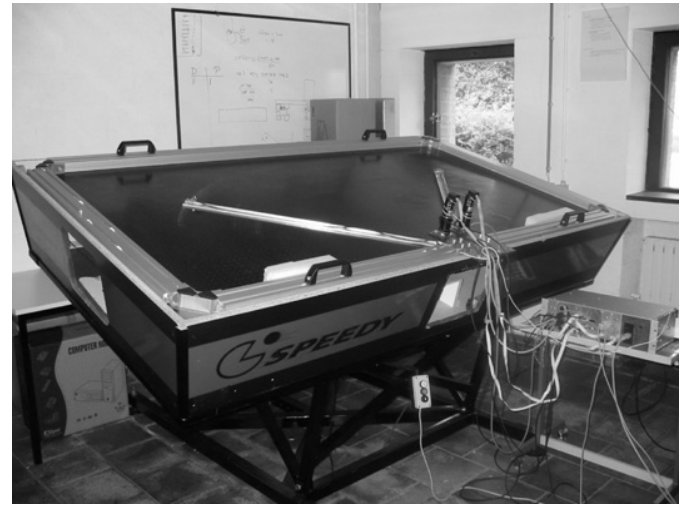

Fig. 2. Picture of Wiper.

\section{Wiper: An experimental setup amenable to mathematical modeling}

Juggling has long been recognized in the literature as a typical coordinated rhythmic task (Beek \& Lewbel, 1995) to study the learning stages of skills' acquisition (Beek \& van Santvoord, 1992) or the use of visual feedback (Huys \& Beek, 2002). The task requires phase-locking relationships between the upper-limbs and the juggled objects trajectories: the total duration of the hands' cycle must equal to the total duration of the balls' cycle (Shannon, 1993). One of the most popular juggling patterns is called the shower, and is depicted in Fig. 1(a): the balls follow a cyclic trajectory along two distinct parabolas produced by a low and a high toss.

We study this steady-state pattern in an experiment that drastically simplifies the hardware: a planar motion of the puck between two impacting edges that idealize the juggler's arms. Fig. 1(b) depicts the so-called period-two orbit of this impact juggler, and the particular period-one orbit (both tosses are equal) is depicted in Fig. 1(c).

Laboratory implementation of this impact juggling experiment has been realized on the Wiper robot, pictured in Fig. 2 .

The motion plane is a tilted air-hockey table. Air-hockey is a popular game which is based on tight goal-shots of plastic pucks on an horizontal table. The puck trajectories are almost frictionless since the table is pierced with a lattice of little holes blowing air constantly. This frictionless table has been tilted with respect to the ground, such that gravity influences the puck motion, like in regular juggling. The two metallic "arms" have a single (rotational) degree of freedom. The pucks have been manufactured from hertalon, a nylon derivative that is both light and elastic.

Wiper is tunable to different configurations (angle of inclination, sensors design, actuation level) and easy to instrument. The edges can indeed be directly actuated by two DC motors, as depicted in Fig. 2. The setup has been used to study the stabilization of simple periodic orbits in impact juggling, such as the period-one and the period-two depicted in Fig. 1. 


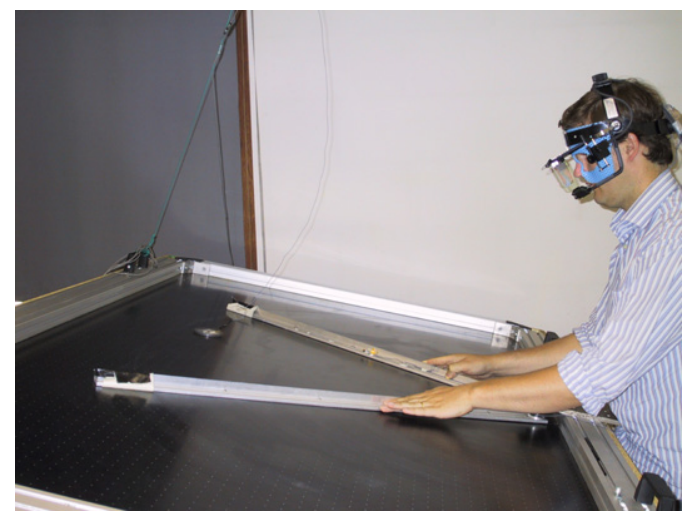

Fig. 3. Wiper can be actuated by human subjects.

Wiper can also be rapidly adapted to study human juggling. Replacing the motors by free rotational joints, human subjects can indeed actuate the edges through direct catching (see Fig. 3).

The stabilization of the period-one juggling task (Fig. 1(c)) is easy and fast to learn. Wiper allows one to test this stabilization task at different tempi, since the flight time between two impacts depends on the steady-state angle between the two edges at impact. If the angle is large, the tempo will be slow, and vice versa. In principle, this setup is also suitable to study more complex patterns, e.g. the period-two (Fig. 1(b)). This would permit one to focus on learning issues and/or feedback selection issues, since the subjects would not be able to keep several pucks in visual tracking at the same time.

Wiper is also amenable to simple mathematical modeling. The dynamical model consists of planar flight phases separated by impacts:

(a) during flight phases, the juggled puck trajectories are ballistic flights along a parabola, solution of Newton's equation $\mathrm{d}^{2} \vec{p} / \mathrm{d} t^{2}=-\vec{g}$ :

$p_{\perp}(t)=p_{\perp}[k]+v_{\perp}[k] t$

$p_{/ /}(t)=p_{/ /}[k]+v_{/ /}[k] t-0.5 g t^{2}$

with $\left(p_{\perp}[k], p_{/ /}[k]\right)$ and $\left(v_{\perp}[k], v_{/ /}[k]\right)$ denoting the position and velocity at impact $k$, orthogonal and parallel to the gravity field, respectively;

(b) at impact, there is a sharp discontinuity in the velocity profile. We model the impact with the simplest Newton's law:

$v_{n}^{+}-\dot{s}_{n}=-e\left(v_{n}^{-}-\dot{s}_{n}\right)$

$v_{t}^{+}-\dot{s}_{t}=v_{t}^{-}-\dot{s}_{t}$

where $\left(v_{n}^{-}, v_{t}^{-}\right)$and $\left(v_{n}^{+}, v_{t}^{+}\right)$are the normal and tangential components of the velocity, with respect to the impacting surface, before and after the impact, respectively; and $\left(\dot{s}_{n}, \dot{s}_{t}\right)$ are the impactor velocity at impact, in the normal and tangential directions w.r.t. the impacting surface.

The coefficient of restitution $0 \leq e \leq 1$ models the dissipated energy at impact. The impact model is only a crude approximate of real impact dynamics, since for example it does not capture spin effects of the puck at impact (Spong, 2001). The complete dynamics of Wiper under these simplifying assumptions has been derived in previous papers (Gerard \& Sepulchre, 2005; Ronsse, Lefevre, \& Sepulchre, 2006, 2007; Sepulchre \& Gerard, 2003).

A further simplification of Wiper's dynamics is of interest to connect the model with the popular 1D bouncing ball model (Bapat, Sankar, \& Popplewell, 1986; Guckenheimer \& Holmes, 1986; Holmes, 1982). Assuming an orthogonal wedge angle and parallel actuations of the edges (that is, the two edges are assumed to remain aligned with the two orthogonal axes of Fig. 4),
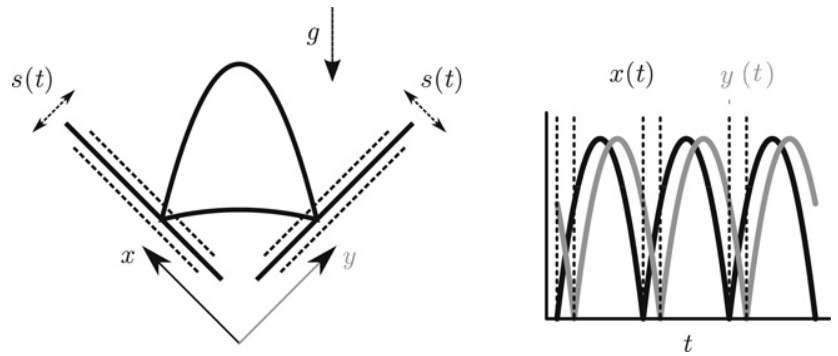

Fig. 4. A special configuration of the wedge planar juggler that decouples the $2 \mathrm{D}$ ball motion (left) into two independent $1 \mathrm{D}$ bouncing ball motions (right). The right frame represents the ball trajectories over time along the $x$ and $y$ axes.

the $2 \mathrm{D}$ motion of the juggled objects projects on each axis to a 1D motion that is unaffected by the bounces on the other axis (Ronsse et al., 2007; Sepulchre \& Gerard, 2003), see Fig. 4.

In this special configuration, a period-two pattern in Wiper corresponds to two frequency-locked period-one bouncing ball patterns (i.e. constant bounce height) along the axes. The phase relationship between those two patterns determines the shape of the periodic orbit. The period-one orbit corresponds to two balls bouncing exactly in anti-phase (i.e. one is at the apex when the other bounces). The period-two patterns correspond to any other phase relationship.

The simplified model (orthogonal wedge with parallel actuation) has thus periodic orbits similar to Wiper. It will be used to capture in a simple way the main properties of these periodic orbits. Further details on the extension to an elaborate model can be found in Ronsse et al. (2006). The present simplified version has been instrumental in developing the feedback control design discussed in the next section.

\section{Rhythmic feedback of Wiper}

\subsection{Sensorless control}

In an unactuated (fixed) and elastic ( $e=1)$ Wiper, the periodic orbits previously described do exist but are not stable (Lehtihet \& Miller, 1986). Real Wiper is obviously not elastic, and requires therefore an actuation to maintain a steady-state pattern. We focused first on open-loop control, i.e. actuation control that is not using any feedback information from the puck state.

Holmes (1982) studied the bouncing ball steady-state periodic orbits under sinusoidal actuation $s(t)=A \sin (\omega t)$. This openloop control provides exponential stability of several periodic obits (including the period-one), depending on the amplitude $A$ and frequency $\omega$ tuning of the sinusoidal actuation profile. We generalized these results to the two-coupled-bouncing-balls Wiper's dynamics - the rotational model developed in Ronsse et al. (2006) - and showed that open-loop sinusoidal actuation of Wiper's edges indeed achieves exponential stability of periodic orbits that are unstable if the wedge is unactuated and elastic. As an illustration, the parametric stability region of the period-one is depicted in Fig. 5.

The period-one stability depends on the amplitude $A$, the coefficient of restitution $e$ and - perhaps less intuitively - on the steady-state impact position $\theta$. The model also predicts the stability of other periodic orbits, under proper tuning of these parameters. Further details can be found in Ronsse et al. (2006).

Direct experimental validations of these theoretical predictions were conducted by actuating Wiper's edges with sinusoidal references. The period-one was successfully stabilized, but steadystate period-two were only observed for a dozen of impacts, in the best cases. Two movies illustrating the successful stabilization of 


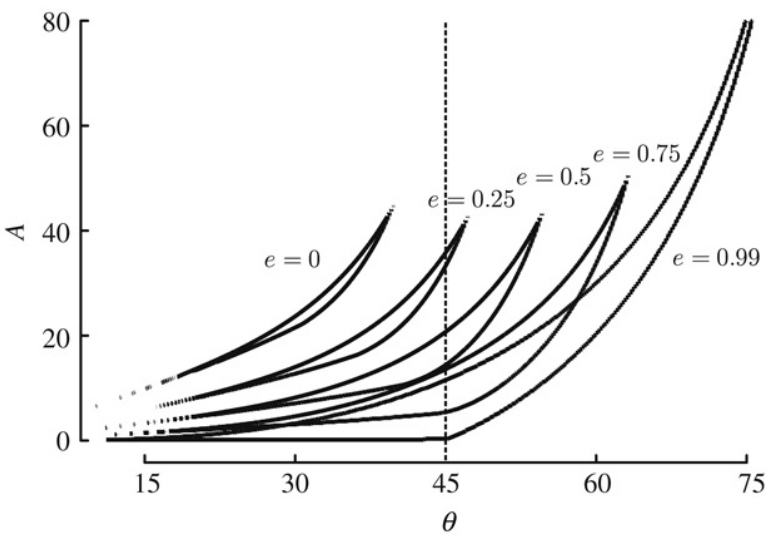

Fig. 5. These curves surround the parametric stability region of Wiper's periodone orbit under open-loop actuation. The parameter space varies with the vibration amplitude $A$ [deg], the coefficient of restitution $e$ and the impact position $\theta$ [deg].

a period-one orbit and the (eventual) failure to stabilize a periodtwo orbit with open-loop actuation are provided as supplementary multimedia materials to Ronsse et al. (2006), as well as on the first author's webpage.

The model derived from parabolic flights and Newton's impact law is only a crude approximation of Wiper's real dynamics. For example, this model does not take into account the variability of the coefficient of restitution along the edges. We also mentioned the puck spin effect that is not accounted for in the model while prominent in period-two trajectories. The basins of attraction predicted by the model therefore ought to be wide enough to compensate for the perturbations induced by these unmodeled dynamics. This was the case for the period-one orbit, but not for the period-two. Stated differently, open-loop stabilization of the period-two orbit is not robust enough in face of the perturbations induced by the variability of the real setup. To enlarge the basins of attraction and make the system more robust, closed-loop control is required.

\subsection{Feedback control}

Buehler, Koditschek, and Kindlmann (1988, 1990, 1994) were the first to address the question of feedback in rhythmic robotic designs, and focused their pioneering investigations on the bouncing ball vertical dynamics. They developed the so-called mirror law algorithms that are based on permanent tracking of the juggled balls and an actuation profile that "mirrors" their motion. A $1 D$ version of the mirror law is given by:

$s(t)=-\frac{1-e}{1+e} \beta(t)-\kappa_{1}\left(\frac{v^{\star 2}}{2}-E(t)\right) \beta(t)$

where $\beta(t)$ and $E(t)$ denote the ball position and energy at time $t$. The first term of (3) mirrors the ball trajectory; the second one is a proportional feedback (gain $=\kappa_{1}$ ) that is used to isolate a particular period-one pattern, characterized by its energy level: $0.5\left(v^{\star}\right)^{2}$, and then that vanishes in steady-state. This feedback control law then requires a continuous tracking of the ball (continuous sensing feedback) that ensures to impact the ball at a constant position $\left(s^{\star}=0\right)$ and with a constant velocity in steady-state. The mirror law and several extensions proved to be very robust, and led to successful experimental validations with bouncing robots in 1D, 2D and 3D environments (Buehler et al., 1988, 1990, 1994).

The mirror law is based on continuous tracking of the juggled objects which requires permanent sensory processing. Given the encouraging results of the open-loop control in our juggling experiment, we investigated the possibility of designing feedback control laws with reduced sensing capabilities. Stated differently, we focused on the minimal feedback that is required to achieve robust closed-loop control, given the fact that sensorless openloop control was already partly satisfactory. Rhythmic impact systems have an exclusive source of feedback that is based on reduced sensing: the measurement of impact times, hence the name of rhythmic feedback control. In impacts systems, the impact times are measured by cheap sensors (e.g. microphones or accelerometers) that record the vibrations induced by the impacts. The measurement of impact times allows one to reconstruct the state of the puck at impact times and, consequently, the entire continuous-time trajectory between impacts, using the solution of (1) (the derivation of this observer is made in Ronsse et al. (2007)).

Our first attempt of feedback control of Wiper was to use the observer to implement a mirror law algorithm of type (3), in which the states $\beta(t)$ and $E(t)$ were replaced by the estimates provided by the observer. This attempt was successful in simulations but miserably failed in the lab, due to the large discrepancy between the reality and the Wiper model.

This prompted us to study the robustness of the closed-loop control with respect to an essential source of uncertainty of the model, i.e. the coefficient of restitution $e$ in the crude impact law (2). The robustness analysis was carried out in Ronsse et al. (2007) on the bouncing ball model for a family of control laws of the form:

$s(t)=s_{u}[k+1]+\dot{s}_{u}[k+1]\left(t-t_{u}[k+1]\right)+\frac{\gamma}{2}\left(t-t_{u}[k+1]\right)^{2}$

where the control parameters $s_{u}[k+1], \dot{s}_{u}[k+1]$, and $t_{u}[k+1]$ are the controller outputs, which are computed on the basis of the input i.e. the impact time $t[k]$ - and the estimated post-impact velocity $\hat{v}[k]$. The free parameter $\gamma$ is the acceleration $\ddot{s}(t)$ at the impact time. Based on the linearized closed-loop dynamics, the transfer function from the coefficient of restitution $e+\delta e[k]$ to the postimpact velocity $v[k]$ is shown to be

$\frac{V(z)}{E(z)}=\frac{2}{1+e} \frac{z+\left(e^{2}+\frac{\gamma}{g}(1+e)^{2}\right)}{z}$

where $V(z)$ and $E(z)$ are the $z$-transforms of $v[k]$ and $\delta e[k]$, respectively. To minimize the dynamical transfer from $\delta e$ to $v$, we selected the acceleration parameter

$\gamma=\frac{-e^{2}}{(1+e)^{2}} g$

which results in a static transfer function. Note that (4) depends on the average coefficient of restitution $e$, which is much easier to estimate than the instantaneous coefficient of restitution $e+\delta e[k]$.

This guiding principle based on elementary system analysis turned out to be the key of success of a laboratory implementation of a rhythmic feedback law. Choice (4) imposes a negative impact acceleration, in contrast to the positive acceleration implied by mirror law algorithms. It is easily shown (Ronsse et al., 2006) that a negative acceleration is also a necessary feature of sensorless control strategies. Our robustness analysis supports previous observations by Schaal et al. (1996) and Sternad et al. (2001a, 2001b). Studying human behavior in a bouncing ball experiment, their results show that subjects consistently hit the ball with negative acceleration on impact. The authors concluded a tendency to rely on open-loop control in such a rhythmic task. Our analysis further confirms that negative acceleration is essential to robustness in limited sensing control strategies.

Since the sinusoidal actuation provides negative acceleration at impact (Schaal et al., 1996; Sternad et al., 2001a, 2001b) and is smooth in steady-state, it was adapted with feedback (Ronsse et al., 2007). Feedback was used to modulate the amplitude and phase from impact-to-impact, such that in steady-state, i.e. when 


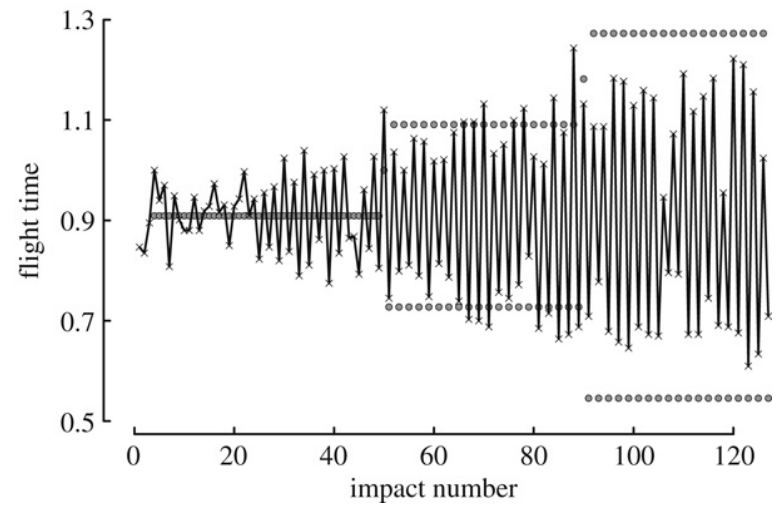

Fig. 6. The flight times between two successive impacts $(t[k]-t[k-1])$ are depicted with the black crosses. The gray circles denote the reference flight times. The first part of the trajectory (about $0<k<50$ ) displays the closed-loop stabilization of a period-one motion: $\Delta t^{\star}=2 v_{\rho}^{\star} / g \simeq 0.91 \mathrm{~s}$. The second part of the trajectory (about $50<k<90$ ) displays the closed-loop stabilization of a "small" period-two motion, i.e. when the reference flight time alternates between $0.9 \Delta t^{\star}$ and $1.1 \Delta t^{\star}$. The last part of the trajectory (about $k>90$ ) displays the closed-loop stabilization of a larger period-two motion: the flight time reference alternates between $0.8 \Delta t^{\star}$ and $1.2 \Delta t^{\star}$.

the corrective feedback signals equal to zero, the actuation is exactly similar to the smooth open-loop profile. This hybrid control scheme provided successful experimental validation for robust stabilization of both period-one and period-two orbits.

Fig. 6 illustrates this experimental validation. It depicts the flight time between two successive impacts, reflecting a transition from period-one to period-two orbit according to the reference. A movie illustrating this successful stabilization of period-one and period-two orbits with closed-loop control based on impact times detection is provided as supplementary multimedia material to Ronsse et al. (2007), as well as on the first author's webpage.

\section{The transition from a discrete to a rhythmic task in human experiments}

We have also used Wiper in behavioral experiments to study the strategies adopted by human subjects in the period-one juggling pattern at different tempi (see Section 2).

We asked nine subjects to juggle the period-one pattern (Fig. 1(c)) at four different tempi, ranging from $\Delta t^{\star}=1000 \mathrm{~ms}$ (the slowest) to $\Delta t^{\star}=400 \mathrm{~ms}$ (the fastest). All the subjects but one were naive with respect to the goals of the experiment. None of them had prior experience with this task. Depending on the tempo, two different typical actuation profiles were observed, the difference of which is illustrated in Fig. 7. At slow tempo, the arms' actuation was intermittent, and strongly decoupled. Observing Fig. 7-top, one can see that both arms were rarely actuated at the same time. In contrast, at fast tempo, the actuation was sustained and strongly synchronized between both arms: their velocity profiles were almost completely in anti-phase (in the egocentric frame of reference), a pattern of actuation that is proved to be reasonably stable (Swinnen, 2002), Fig. 7-bottom. The intermittent behavior can then be viewed as a train of "discrete" strokes, with marked pose intervals in between; and the sustained behavior as a "rhythmic" actuation (Hogan \& Sternad, 2007).

Fig. 8 reveals how the transition took place depending on the tempo. This figure depicts the histograms of the so-called synchronization index of both arms during flight times. This index is equal to the averaged phase relationship between the edges that was computed through the non-normalized correlation coefficient of the right arm velocity $\dot{\theta}_{r}$ and the left arm velocity $\dot{\theta}_{l}$ :

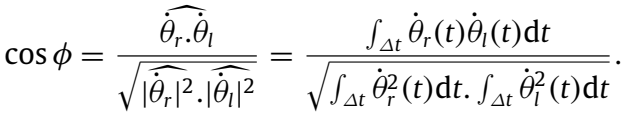
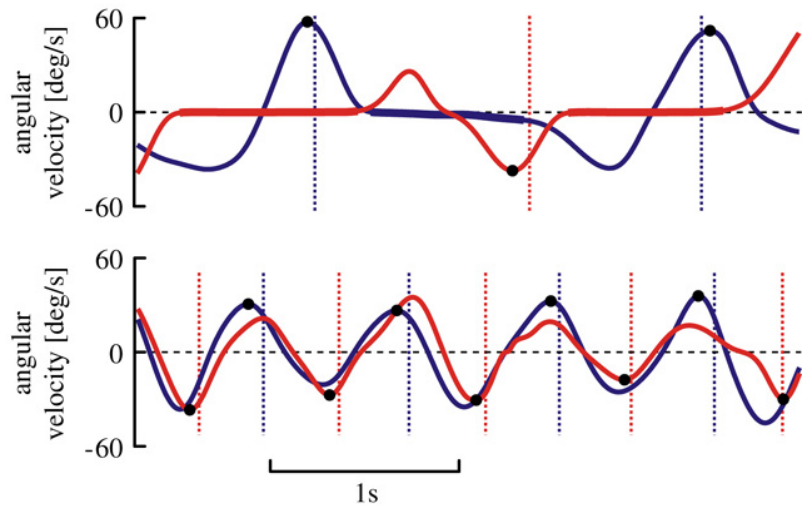

Fig. 7. Typical angular velocity profiles of Wiper's edges at the slowest (top, $\Delta t^{\star}=1000 \mathrm{~ms}$ ) and the fastest (bottom, $\Delta t^{\star}=400 \mathrm{~ms}$ ) tempi. The right and left arm velocity profiles are represented in red and blue, respectively. The thicker portions emphasize the periods where the arms were at rest. The vertical lines denote the impact times. The black dots denote the point of maximum velocity around impacts. (For interpretation of the references to colour in this figure legend, the reader is referred to the web version of this article.)
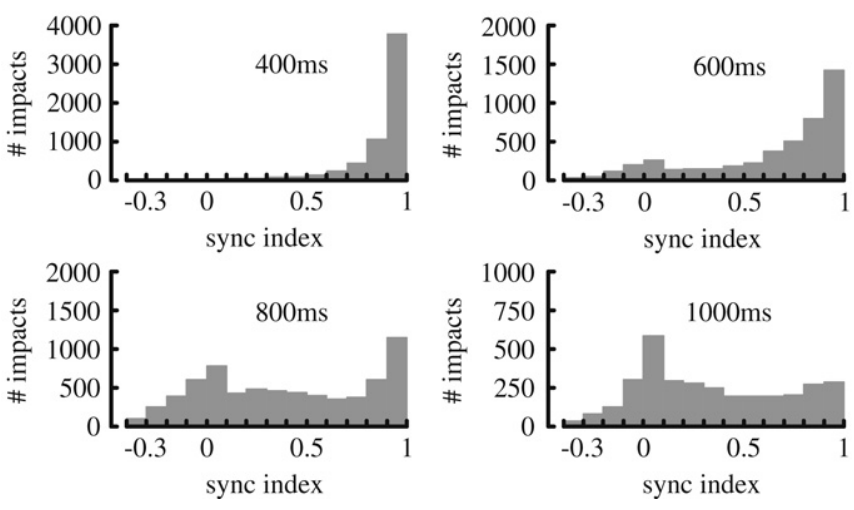

Fig. 8. Histograms of the synchronization index, depending on the tempo $\Delta t^{\star}$.

At the fastest tempo ( $\left.\Delta t^{\star}=400 \mathrm{~ms}\right)$, the arms were almost all the time synchronized $(\cos \hat{\phi} \simeq 1)$ for the nine tested subjects. As the tempo decreased, the second strategy (decoupled actuation, $\cos \hat{\phi} \simeq 0$ ) emerged, since more and more subjects passed their transition level.

We also investigated how these two modes of control relied on feedback processing. During separate recording blocks, our subjects were asked to perform the task by closing their eyes once in steady-state. The influence of visual feedback availability is illustrated in Fig. 9. The black line reproduces the average values of the histograms depicted in Fig. 8, i.e. the transition from a strongly synchronized actuation mode to a decoupled mode as the tempo decreased. The gray line refers to the synchronization index for the data without visual feedback. The upward shift from the black curve to the gray curve reveals a tendency of the subjects to favor the synchronized behavior when deprived of their visual feedback. Both the absence of visual feedback and the fast tempo reduce the role of the sensorimotor loop and favor the rhythmic behavior.

\section{Conclusion}

This paper puts in a unique perspective recent theoretical and experimental work done by the authors on a simple rhythmic task. Understanding the role of feedback in this setting is of interest both in robotics and in neuroscience. Juggling has long been recognized as a benchmark for rhythmic tasks, requiring the coordination between the upper-limbs to stabilize a broad set of periodic patterns. The proposed impact juggler, called Wiper, shares these 


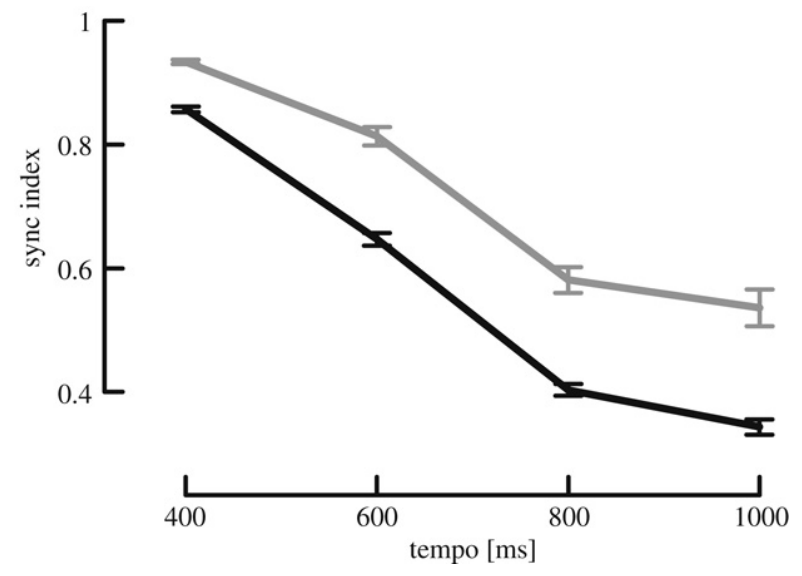

Fig. 9. Synchronization index depending on the tempo $\Delta t^{\star}$. The black lines depict the impacts with visual feedback while the gray lines those without visual feedback. Mean \pm 0.95 Conf. Interval. A factorial ANOVA $(4 \times 2)$ revealed this parameter dependence on the tempo, on the visual feedback condition and on their interaction (all $p<0.05$ ).

features but is amenable to simpler mathematical modeling, based on a $2 \mathrm{D}$ generalization of the popular $1 \mathrm{D}$ bouncing ball.

In Section 3, we detailed the theoretical and experimental results obtained in robotics control of Wiper's periodic orbits (Gerard \& Sepulchre, 2005; Ronsse et al., 2006, 2007; Sepulchre \& Gerard, 2003). Sensorless open-loop control is efficient for the simplest juggling pattern, revealing a $2 \mathrm{D}$ analogy with the stable bouncing ball under sinusoidal actuation (Bapat et al., 1986; Guckenheimer \& Holmes, 1986; Holmes, 1982). However, when considering more complex periodic patterns, the predicted basin of attraction of the stabilized orbit is small and the experimental implementation is not robust, due to large uncertainties of the real setup not accounted for in the model. Stated differently, the open-loop actuation was not robust enough with respect to the unmodeled dynamics. The basins of attraction have been enlarged by modulating the open-loop trajectory through limited feedback provided by measuring only the times of impact.

Interestingly, a robustness analysis revealed that a closed-loop actuation resting on this limited source of feedback is robust if its acceleration has a particular negative value at impact. This nicely connects with the open-loop actuation since the openloop profile has a negative acceleration at impact in steadystate. Schaal et al. (1996) and Sternad et al. (2001a, 2001b) reported furthermore that humans produce a similar actuation trajectory (with negative acceleration at impact) in a 1D bouncing task. These authors hypothesized that this negative acceleration in human data is a signature of the exploitation of the open-loop dynamical properties, despite recent evidences of active closed-loop control in a similar protocol (de Rugy, Wei, Muller, \& Sternad, 2003; Wei, Dijkstra, \& Sternad, 2007). Our analysis further suggests that negative acceleration at impact is not restricted to open-loop strategies but a crucial feature of robust closed-loop control based on limited feedback.

We also analyzed the behavior of human subjects when juggling with Wiper (see Section 4). Human subjects switched their actuation strategy from a train of discrete actuations to a rhythmic actuation depending on the task tempo. Our observations supported the existence of two fundamental modes of control (or "primitives"), with clearly distinct sensory feedback requirements (Ronsse, Thonnard, Lefevre, \& Sepulchre, in press). Interestingly, the intermittent type of control recruited in our task is very similar to the type of control for postural sway (see e.g. Bottaro, Casadio, Morasso, and Sanguineti (2005), Loram, Gawthrop, and Lakie (2006) and Loram and Lakie (2002) and stick balancing at the fingertip Cabrera and Milton (2002, 2004).

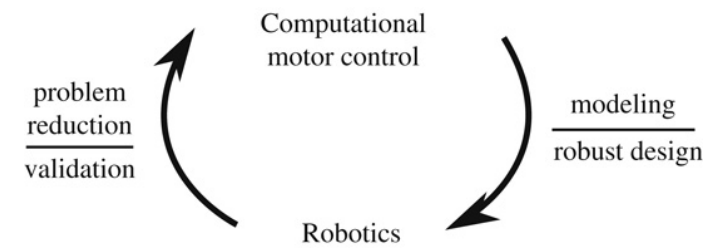

Fig. 10. Experimental results in computational motor control neuroscience and robotics cross-fertilize.

These two intrinsically unstable tasks are indeed controlled by a blend of passive control (under the unstable dynamics) and active compensation at periodic time intervals. The "Act-and-Wait" control framework proposed by Insperger (2006) emphasizes why such strategies permit the tuning of low-gain controllers for unstable systems in the presence of significant delays in the loop (see also Mehta and Schaal (2002), for the pole balancing control). Wiper can be used as a modeling and experimental benchmark in this framework, and the results described in the present paper naturally invite one to investigate the relevance of rhythmic feedback - i.e. based on timed events in cycle - designs for such skilled performances.

As illustrated in Fig. 10, this study took advantage of the interaction between computational motor control neuroscience and robotics. The mathematical modeling of the task aimed at reducing the problem complexity by emphasizing a limited number of relevant parameters, being useful to analyze the behavior and discriminate between different control strategies. It provides a direct comparison with the theoretical predictions and robotics implementations. Finally, the data suggested that humans use mainly two distinct primitives to control rhythmic tasks (Schaal \& Schweighofer, 2005), i.e. an intermittent train of discrete strokes or a sustained rhythmic movement. This might inspire a flexible computational model to generate complex movements, based on simple interactions between fundamental primitives.

\section{Acknowledgements}

The authors would like to thank the anonymous reviewers for their helpful comments that improved the paper.

This paper presents research results of the Belgian Network DYSCO (Dynamical Systems, Control, and Optimization), funded by the Interuniversity Attraction Poles Programme, initiated by the Belgian State, Science Policy Office. The scientific responsibility rests with its authors. P. Lefèvre is funded by the 'Fonds National de la Recherche Scientifique'; the 'Fondation pour la Recherche Scientifique Médicale'; an internal research grant ('Fonds Spéciaux de Recherche') from the 'Université catholique de Louvain'; the European Space Agency (EU); and the PRODEX program (Belgium).

\section{References}

Bapat, C., Sankar, S., \& Popplewell, N. (1986). Repeated impacts on a sinusoidally vibrating table. Journal of Sound and Vibration, 108(1), 99-115.

Beek, P. J., \& Lewbel, A. (1995). The science of juggling. Scientific American, 273(5), 92-97.

Beek, P. J., \& van Santvoord, A. A. M. (1992). Learning the cascade juggle - a dynamicsystems analysis. Journal of Motor Behaviour, 24(1), 85-94.

Bernstein, N. (1967). The coordination and regulation of movements. London: Pergamon.

Bottaro, A., Casadio, M., Morasso, P. G., \& Sanguineti, V. (2005). Body sway during quiet standing: Is it the residual chattering of an intermittent stabilization process?. Human Movement Science, 24(4), 588-615.

Buchanan, J. J., Park, J. H., \& Shea, C. H. (2006). Target width scaling in a repetitive aiming task: Switching between cyclical and discrete units of action. Experimental Brain Research, 175(4), 710-725.

Buehler, M., Koditschek, D., \& Kindlmann, P. (1988). A one degree of freedom juggler in a two degree of freedom environment. In: IEEE/RSJ conf. intelligent systems and robots (pp. 91-97). 
Buehler, M., Koditschek, D., \& Kindlmann, P. (1990). A family of robot control strategies for intermittent dynamical environments. IEEE Control Systems Magazine, 10(2), 16-22.

Buehler, M., Koditschek, D., \& Kindlmann, P. (1994). Planning and control of robotic juggling and catching tasks. International Journal of Robotics Research, 13(2), 101-118.

Cabrera, J. L., \& Milton, J. G. (2002). On-off intermittency in a human balancing task. Physical Review Letters, 89(15), 158702.

Cabrera, J. L., \& Milton, J. G. (2004). Human stick balancing: Tuning levy flights to improve balance control. Chaos, 14(3), 691-698.

Cohen, A., Rossignol, S., \& Grillner, S. (1988). Neural control of rhythmic movements in vertebrates. New York: Wiley.

Collins, J. J., \& Richmond, S. A. (1994). Hard-wired central pattern generators for quadrupedal locomotion. Biological Cybernetics, 71(5), 375-385.

Collins, S., Ruina, A., Tedrake, R., \& Wisse, M. (2005). Efficient bipedal robots based on passive-dynamic walkers. Science, 307(5712), 1082-1085.

Collins, S. H., Wisse, M., \& Ruina, A. (2001). A three-dimensional passive-dynamic walking robot with two legs and knees. International Journal of Robotics Research, 20(7), 607-615.

de Rugy, A., Wei, K., Muller, H., \& Sternad, D. (2003). Actively tracking 'passive' stability in a ball bouncing task. Brain Research, 982(1), 64-78.

Desmurget, M., Grea, H., Grethe, J. S., Prablanc, C., Alexander, G. E., \& Grafton, S. T. (2001). Functional anatomy of nonvisual feedback loops during reaching: positron emission tomography study. Journal of Neuroscience, 21(8), 2919-2928.

Duysens, J., \& Van de Crommert, H. W. (1998). Neural control of locomotion; the central pattern generator from cats to humans. Gait \& Posture, 7(2), 131-141.

Gerard, M., \& Sepulchre, R. (2005). Rhythmic stabilization of periodic orbits in a wedge. In P. Horacek, M. Simandl, \& P. Zitek (Eds.), IFAC world congress. Czech Republic: Prague.

Guckenheimer, J., \& Holmes, P. J. (1986). Nonlinear oscillations, dynamical systems and bifurcations of vector fields. New York: Springer-Verlag.

Haruno, M., Wolpert, D. M., \& Kawato, M. (2001). Mosaic model for sensorimotor learning and control. Neural Computation, 13(10), 2201-2220.

Hogan, N., \& Sternad, D. (2007). On rhythmic and discrete movements: Reflections, definitions and implications for motor control. Experimental Brain Research, 181(1), 13-30.

Holmes, P. J. (1982). The dynamics of repeated impacts with a sinusoidally vibrating table. Journal of Sound and Vibration, 84(2), 173-189.

Huys, R., \& Beek, P. J. (2002). The coupling between point-of-gaze and ball movements in three-ball cascade juggling: The effects of expertise, pattern and tempo. Journal of Sports Sciences, 20(3), 171-186.

Insperger, T. (2006). Act-and-wait concept for continuous-time control systems with feedback delay. IEEE Transactions on Control Systems Technology, 14(5), 974-977.

Jordan, M. I., \& Wolpert, D. M. (1999). Computational motor control. In M. Gazzaniga (Ed.), The cognitive neurosciences. Cambridge, MA: MIT Press.

Kawato, M. (1999). Internal models for motor control and trajectory planning. Current Opinion in Neurobiology, 9(6), 718-727.

Kelso, J. A. (1984). Phase transitions and critical behavior in human bimanual coordination. American Journal of Physiology, 246(6 Pt 2), R1000-4.

Kelso, J. A. S. (1995). Dynamic patterns. In The self-organization of brain and behavior. Cambridge, MA: MIT Press.

Kelso, J. A. S., Southard, D. L., \& Goodman, D. (1979). On the nature of human interlimb coordination. Science, 203(4384), 1029-1031.

Kuo, A. D. (1999). Stabilization of lateral motion in passive dynamic walking International Journal of Robotics Research, 18(9), 917-930.

Lehtihet, H. E., \& Miller, B. N. (1986). Numerical study of a billiard in a gravitational field. Physica D, 21(1), 93-104.

Loram, I. D., Gawthrop, P. J., \& Lakie, M. (2006). The frequency of human, manual adjustments in balancing an inverted pendulum is constrained by intrinsic physiological factors. Journal of Physiology, 577(Pt 1), 417-432.

Loram, I. D., \& Lakie, M. (2002). Human balancing of an inverted pendulum: Position control by small, ballistic-like, throw and catch movements. Journal of Physiology, 540(Pt 3), 1111-1124.

Mehta, B., \& Schaal, S. (2002). Forward models in visuomotor control. Journal of Neurophysiology, 88(2), 942-953.
Miall, R., Weir, D., Wolpert, D., \& Stein, J. (1993). Is the cerebellum a smith predictor? Journal of Motor Behaviour, 25(3), 203-216.

Puttemans, V., Wenderoth, N., \& Swinnen, S. P. (2005). Changes in brain activation during the acquisition of a multifrequency bimanual coordination task: From the cognitive stage to advanced levels of automaticity. Journal of Neuroscience, 25(17), 4270-4278.

Ronsse, R., Lefevre, P., \& Sepulchre, R. (2006). Sensorless stabilization of bounce juggling. IEEE Transactions on Robotics, 22(1), 147-159.

Ronsse, R., Lefevre, P., \& Sepulchre, R. (2007). Rhythmic feedback control of a blind planar juggler. IEEE Transactions on Robotics, 23(4), 790-802.

Ronsse, R., Thonnard, J.-L., Lefevre, P., \& Sepulchre, R. (2008). Control of bimanual rhythmic movements: Trading efficiency for robustness depending on the context. Experimental Brain Research, 187(2), 193-205. http://dx.doi.org/10.1007/s00221-008-1297-9.

Schaal, S., Atkeson, C. G., \& Sternad, D. (1996). One-handed juggling: A dynamical approach to a rhythmic movement task. Journal of Motor Behaviour, 28(2), 165-183.

Schaal, S., \& Schweighofer, N. (2005). Computational motor control in humans and robots. Current Opinion in Neurobiology, 15(6), 675-682.

Schaal, S., Sternad, D., Osu, R., \& Kawato, M. (2004). Rhythmic arm movement is not discrete. Nature Neuroscience, 7(10), 1136-1143.

Scott, S. H. (2004). Optimal feedback control and the neural basis of volitional motor control. Nature Reviews Neuroscience, 5(7), 532-546.

Scott, S. H., \& Norman, K. E. (2003). Computational approaches to motor control and their potential role for interpreting motor dysfunction. Curr Opin Neurol, 16(6), 693-698.

Sepulchre, R., \& Gerard, M. (2003). Stabilization of periodic orbits in a wedge billiard. In D. A. Lawrence, \& T. Parisini (Eds.), 42nd IEEE conference on decision and control (pp. 1568-1573). Hawaii: IEEE.

Shadmehr, R., \& Wise, S. P. (2005). The computational neurobiology of reaching and pointing. A foundation for motor learning. Cambridge, MA: MIT Press.

Shannon, C. E. (1993). Scientific aspects of juggling. In N. J. A. Sloane, \& A. D. Wyner (Eds.), Claude Elwood Shannon: Collected papers (p. 924). New York: IEEE Press.

Spong, M. W. (2001). Impact controllability of an air hockey puck. Systems and Control Letters, 42(5), 333-345.

Sternad, D., Dean, W. J., \& Schaal, S. (2000). Interaction of rhythmic and discrete pattern generators in single-joint movements. Human Movement Science, 19(4), 627-664.

Sternad, D., Duarte, M., Katsumata, H., \& Schaal, S. (2001a). Bouncing a ball: Tuning into dynamic stability. Journal of Experimental Psychology - Human Perception and Performance, 27(5), 1163-1184.

Sternad, D., Duarte, M., Katsumata, H., \& Schaal, S. (2001b). Dynamics of a bouncing ball in human performance. Physical Review E, 6301(1), art. no.-011902.

Strogatz, S. H. (2003). Sync: The emerging science of spontaneous order. New York: Hyperion.

Swinnen, S. P. (2002). Intermanual coordination: From behavioural principles to neural-network interactions. Nature Reviews Neuroscience, 3(5), 348-359.

Swinnen, S. P., \& Wenderoth, N. (2004). Two hands, one brain: Cognitive neuroscience of bimanual skill. Trends in Cognitive Sciences, 8(1), 18-25.

Todorov, E. (2004). Optimality principles in sensorimotor control. Nature Neuroscience, 7(9), 907-915.

Todorov, E., \& Jordan, M. I. (2002). Optimal feedback control as a theory of motor coordination. Nature Neuroscience, 5(11), 1226-1235.

Turvey, M. T. (1990). Coordination. American Psychologist, 45(8), 938-953.

van Mourik, A. M., \& Beek, P. J. (2004). Discrete and cyclical movements: Unified dynamics or separate control?. Acta Psychologica (Amst), 117(2), 121-138.

Wei, K., Dijkstra, T. M. H., \& Sternad, D. (2007). Passive stability and active control in a rhythmic task. Journal of Neurophysiology, 98, 2633-2646. http://dx.doi.org/10.1152/jn.00742.2007.

Wolpert, D., \& Miall, R. (1996). Forward models for physiological motor control. Neural Networks, 9(8), 1265-1279.

Wolpert, D. M., \& Ghahramani, Z. (2000). Computational principles of movement neuroscience. Nat Neurosci 3 Suppl, 1212-1217.

Wolpert, D. M., Miall, R. C., \& Kawato, M. (1998). Internal models in the cerebellum. Trends Cognition Science, 2(9), 338-347. 